SLAC-PUB-8028

Revised March 2006

\title{
EFFECT OF CENTRIFUGAL TRANSVERSE WAKEFIELD FOR MICROBUNCH IN BEND*
}

\author{
G.V. Stupakov \\ Stanford Linear Accelerator Center, Stanford University, Stanford, CA 94309
}

\begin{abstract}
We calculate centrifugal force for a short bunch in vacuum moving in a circular orbit and estimate the emittance growth of the beam in a bend due to this force.
\end{abstract}

Presented at the 16th ICFA Beam Dynamics Workshop on Nonlinear and Collective Phenomena in Beam Physics, Arcidosso, Italy, September 1, 1998 - September 5, 1998. 


\title{
Effect of Centrifugal Transverse Wakefield for Microbunch in Bend
}

\author{
G. V. Stupakov \\ Stanford Linear Accelerator Center Stanford University, Stanford, CA 94309
}

\begin{abstract}
We calculate centrifugal force for a short bunch in vacuum moving in a circular orbit and estimate the emittance growth of the beam in a bend due to this force.
\end{abstract}

\section{INTRODUCTION}

Many of the basic features of the coherent synchrotron radiation (CSR) of short bunches and its effect on beam dynamics in accelerators are now well established [1-4]. The effect is usually described in terms of the longitudinal force, or wakefield, that causes the energy loss in the beam, and also redistributes the energy between the particles by accelerating the head and decelerating the tail of the bunch. Coherent radiation becomes most important for short bunches and high currents. More subtle features of CSR such as transition effect due to the entrance to and exit from the bend [5], CSR force in the undulator [6], and shielding due to the close metallic boundaries [7] have been also studied.

Much less is known about the transverse force in a short bunch moving on a circular orbit. The problem has been treated in several papers beginning from $\mathrm{R}$. Talman's work [8], who pointed out that the centrifugal force of a rotating bunch can result in a noticeable tune shift of betatron oscillations. Later, an important correction to the Talman paper has been added in Ref. [9], where it was shown that due to the energy variation in the bunch, the effect of the transverse force proportional to $R^{-1}$ is cancelled, and the residual effect is of the order of $R^{-2}$, that is much smaller than originally predicted. Recently, however, Derbenev and Shiltsev [10] found the centrifugal force of the order of $R^{-1}$ that differs from Talman's result by a logarithmic factor only.

Taking into account the existing controversy in the literature, in this paper, we consider the transverse force in a bunch based on simple physical arguments, starting from a dc beam. We will derive the centrifugal force for a relativistic coasting beam in vacuum, and then generalize the result for a short bunch, and estimate its effect on the emittance growth in a bend. 
Throughout this paper we assume ultrarelativistic beam, $v=c$, moving on a circular orbit of radius $R$.

\section{LIENARD-WIECHERT POTENTIALS AND FIELDS}

The electromagnetic field of a point charge moving in vacuum, as is well known, can be found using Lienard-Wiechert potentials, and the fields can be explicitly expressed in terms of particle's velocity and acceleration at the retarded time [11]. We will use the coordinate system shown in Fig. 1. For $\gamma=\infty$, the fields of the

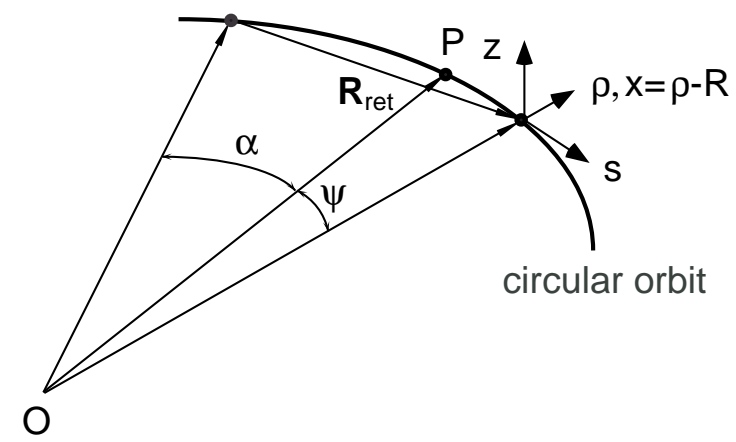

FIGURE 1. Coordinate system. Shown are locations of the particle at time $t$, the point $\mathrm{P}$, the observation point in front of the particle at an angle $\psi$, and the point where the radiation occurred at the retarded time (at an angle $\alpha$ behind the particle). Vector $\boldsymbol{R}_{\text {ret }}$ connects the radiation point with the observation point.

charge are given by the following equations,

$$
\begin{gathered}
\boldsymbol{E}=\frac{q}{c} \frac{\boldsymbol{R}_{\mathrm{ret}} \times\left[\left(\boldsymbol{R}_{\mathrm{ret}}-R_{\mathrm{ret}} \boldsymbol{\beta}_{\mathrm{ret}}\right) \times \dot{\boldsymbol{\beta}}_{\mathrm{ret}}\right]}{\left(\boldsymbol{R}_{\mathrm{ret}}-R_{\mathrm{ret}} \boldsymbol{\beta}_{\mathrm{ret}}\right)^{3}}, \\
\boldsymbol{H}=\frac{1}{R_{\mathrm{ret}}}\left[\boldsymbol{E} \times \boldsymbol{R}_{\mathrm{ret}}\right],
\end{gathered}
$$

where the distance $\boldsymbol{R}_{\text {ret }}$ shown in Fig. 1 connects the position of the particle at the radiation time $t_{\text {ret }}$ and the observation point, $R_{\text {ret }}=c\left(t-t_{\text {ret }}\right), \boldsymbol{\beta}_{\text {ret }}$ is the unit vector $(v=c)$ directed along the particle velocity at the radiation time, and $\dot{\boldsymbol{\beta}}_{\text {ret }}$ is the derivative of the velocity at that time.

If the transverse size of the bunch $\sigma_{r}$ is much smaller than its length $\sigma_{z}$, only the field on the orbit interacts with the beam. In this case, the observation point can be chosen on the circle, as shown in Fig. 1, and Eqs. (1) and (2) can be written as (see Fig. 1 for notation) 


$$
\begin{aligned}
& E_{s}(\psi)=\frac{q}{R^{2}} \frac{2 \sin \frac{1}{2}(\alpha+\psi)}{[\alpha-\sin (\alpha+\psi)]^{3}} \\
& \times\left\{2\left(\sin \frac{1}{2}(\alpha+\psi)\right)^{3}\left[\cos \frac{1}{2}(\alpha+\psi)-\cos (\alpha+\psi)\right]\right. \\
&-\sin (\alpha+\psi)[\alpha-\sin (\alpha+\psi)]\}, \\
& E_{\rho}(\psi)=\frac{q}{R^{2}} \frac{2 \sin \frac{1}{2}(\alpha+\psi)}{[\alpha-\sin (\alpha+\psi)]^{3}} \\
& \times\left\{2\left(\sin \frac{1}{2}(\alpha+\psi)\right)^{3}\left[\sin \frac{1}{2}(\alpha+\psi)-\sin (\alpha+\psi)\right]\right. \\
&+\cos (\alpha+\psi)[\alpha-\sin (\alpha+\psi)]\}, \\
& H_{z}(\psi)=E_{s} \sin \frac{1}{2}(\alpha+\psi)-E_{\rho} \cos \frac{1}{2}(\alpha+\psi),
\end{aligned}
$$

where $E_{s}(\psi)$ is the longitudinal and $E_{\rho}(\psi)$ - radial components of the electric field, $H_{z}(\psi)$ is the vertical magnetic field, $\psi=s / R$, and the angle $\alpha$ is related to the position of the observation point by equation

$$
\alpha=2\left|\sin \frac{1}{2}(\psi+\alpha)\right| .
$$

The plot of the longitudinal field as a function of the position on the circle is shown in Fig. 2.

The electric field $E_{s}$ per unit charge is equal to the longitudinal wake $w$. For small distances, $s \ll R$, one finds from Eqs. (3) and (6),

$$
w(s)=\frac{1}{q} E_{s}(s) \approx\left\{\begin{array}{cc}
2(3 s)^{-4 / 3} R^{-2 / 3}, & s>0 \\
0, & s<0 .
\end{array}\right.
$$

For a short bunch $\left(\sigma_{z} \ll R\right)$ with a given charge distribution $\lambda(s)\left(\int \lambda(s) d s=1\right)$, the longitudinal wake for the bunch is defined as a convolution with the distribution function,

$$
\begin{aligned}
& w_{\text {bunch }}(s)=\int_{-\infty}^{\infty} w\left(s-s^{\prime}\right) \lambda\left(s^{\prime}\right) d s^{\prime} \\
= & \frac{2}{3^{3 / 4} R^{2 / 3}} \int_{s}^{\infty} \frac{\lambda\left(s^{\prime}\right) d s^{\prime}}{\left(s^{\prime}-s\right)^{4 / 3}} .
\end{aligned}
$$

The problem here is that the above integral diverges when $s^{\prime} \rightarrow s$. To overcome this difficulty, we can use a trick and integrate the last equation by parts (neglecting the nonintegral term!):

$$
w_{\text {bunch }}(s)=\frac{2}{3^{1 / 4} R^{2 / 3}} \int_{0}^{\infty} \frac{d s^{\prime}}{\left(s^{\prime}-s\right)^{1 / 3}} \frac{d \lambda\left(s^{\prime}\right)}{d s^{\prime}} .
$$




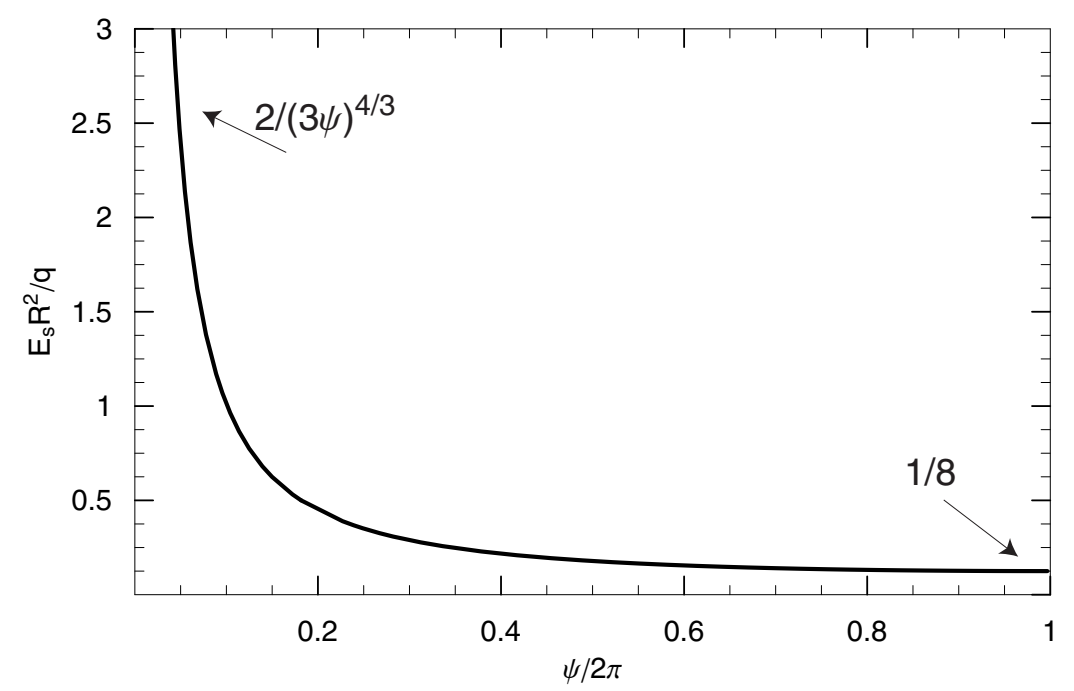

FIGURE 2. Longitudinal electric field on a circular orbit as a function of angle $\psi$ counted from the location of the particle. The arrows show asymptotic expressions for the field in the vicinity of the particle.

Now the integral converges, and gives the right result for the wake $[2,3]$. The justification for this trick can be found in a more accurate consideration of the fields in a small vicinity of the particle $[4,5]$.

We also mention here that although we obtained the above result assuming an infinitely thin beam, the applicability condition for the longitudinal wake is actually very mild , $\sigma_{r} / \sigma_{z} \ll\left(R / \sigma_{z}\right)^{1 / 3}[3]$. At this point we are tempted to apply the same approach for the calculation of the transverse force in a thin bunch. The transverse force (per unit charge) $F_{\rho}$ for an ultrarelativistic bunch is $F_{\rho}=E_{\rho}+H_{z}$. The plots of $E_{\rho}$ and $H_{z}$ are shown in Fig. 3, and the transverse force as a function of angle $\psi$ on the circular orbit is shown in Fig. 4. Asymptotically, for small positive $\psi$ in front of the particle $F_{\rho} \approx q / 3 R^{2} \psi$; behind the particle, for negative small $\psi, F_{\rho} \approx q / R^{2}|\psi|$. Again, if we want to convolve this force with the bunch distribution and to find the transverse wakefield for the bunch, as we did above for the longitudinal wake, the integral would diverge, and there is no trick that could make it convergent. As we will see in the next section, there is a profound reason for such divergence: the transverse force depends on the beam radius $\sigma_{r}$ that we neglected in the above consideration.

\section{TRANSVERSE FORCE - COASTING BEAM}

To make our consideration of the transverse force as simple as possible we begin here from a problem of a coasting relativistic dc beam of radius $a$, shown in Fig. 5. To find the transverse force $f_{\rho}$ acting on a unit length of the beam in this case, 


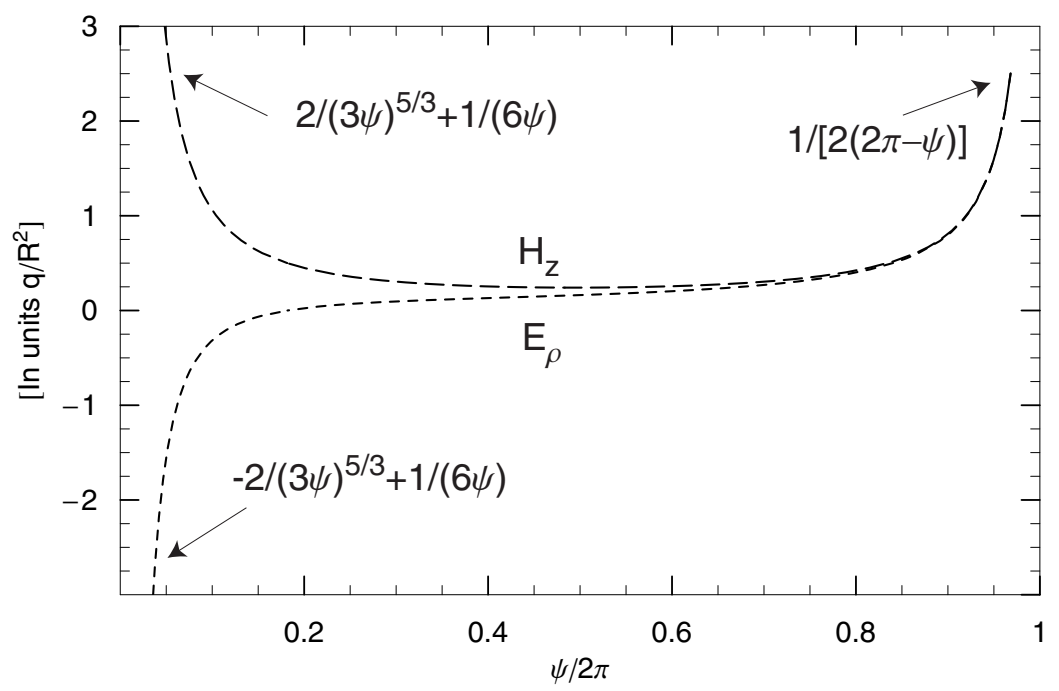

FIGURE 3. Radial electric field $E_{\rho}$ and vertical magnetic field $H_{z}$ on the orbit. Arrows show the asymptotic expressions for the fields near the particle.

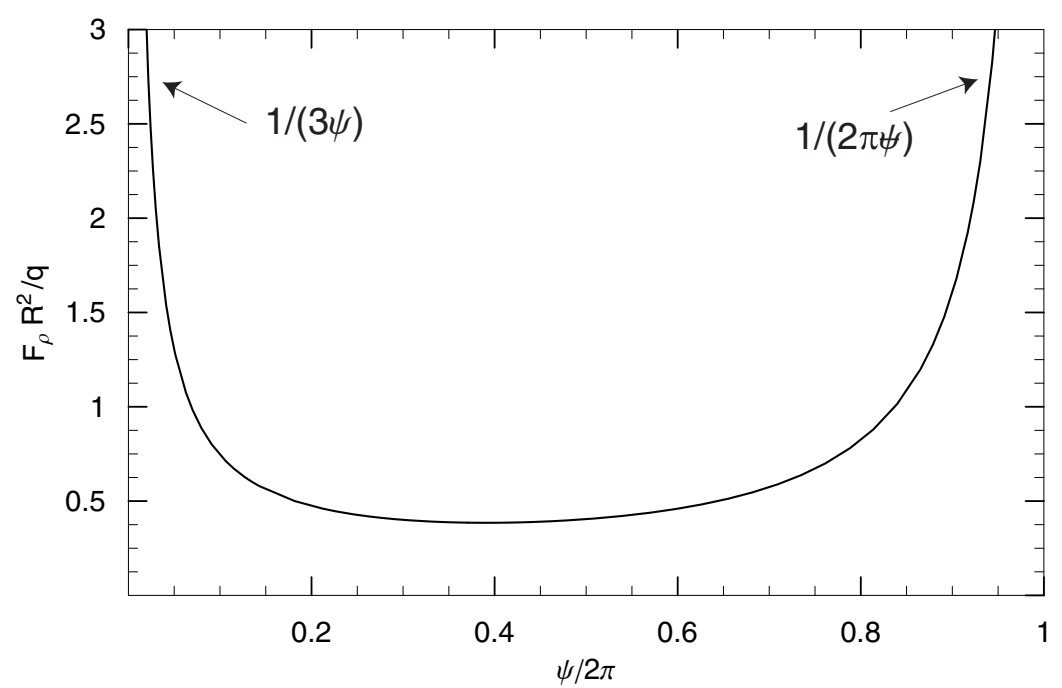

FIGURE 4. Transverse force per unit charge $F_{\rho}$ as a function of angle $\psi$. Arrows show asymptotic expressions for the force near the particle.

we will use the energy principle that relates the force to the variation of the energy of the system under infinitesimally small displacement [12]. Since the electric and magnetic fields of a coasting beam are time independent, the electromagnetic energy of the beam is the sum of the electrostatic and magnetic energies. To find them, we need to know the capacitance and inductance of a charged rotating ring, which can be found in textbooks on electrodynamics (see, e.g., [12]). 


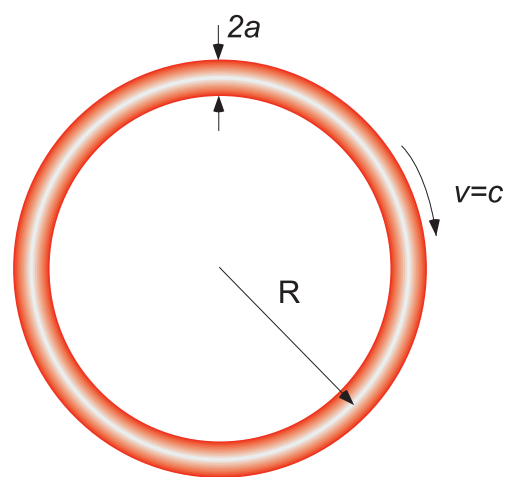

FIGURE 5. Coasting beam of radius a moving along a circular orbit in vacuum.

The inductance of a circular current in vacuum is given by the following formula,

$$
L=4 \pi R\left(\ln \frac{8 R}{a}-\frac{7}{4}\right) .
$$

Differentiating the magnetic energy $L I^{2} / 2 c^{2}$ with respect to the circumference of the beam, we obtain the radial magnetic force per unit length of the ring,

$$
f_{m}=\frac{\partial\left(L I^{2} / 2 c^{2}\right)}{\partial(2 \pi R)}=\frac{I^{2}}{R c^{2}}\left(\ln \frac{8 R}{a}-\frac{3}{4}\right) .
$$

Analogously, the capacitance $C$ of the uniformly charged ring is

$$
C^{-1}=\frac{1}{\pi R}\left(\ln \frac{8 R}{a}+\frac{1}{4}\right)
$$

and the electric force per unit length is equal to the derivative of the electrostatic energy with respect to the circumference (with the minus sign),

$$
f_{e}=-\frac{\partial\left(Q^{2} / 2 C\right)}{\partial(2 \pi R)}=\frac{Q^{2}}{4 \pi^{2} R^{3}}\left(\ln \frac{8 R}{a}-\frac{3}{4}\right) .
$$

Taking into account that for an ultrarelativistic beam $Q=2 \pi R I / c$, we find that the electric and magnetic forces are equal, which is expected in the limit $v=c$.

Adding Eq. (12) and (14) gives the total force,

$$
f_{\rho}=f_{m}+f_{e}=\frac{I^{2}}{R c^{2}}\left(2 \ln \frac{8 R}{a}-\frac{3}{2}\right) .
$$

We can also easily find the force, if the beam propagates in a circular pipe of radius $b$. In this case the capacitance of the ring is,

$$
C^{-1}=\frac{1}{\pi R}\left(\ln \frac{b}{a}+\frac{1}{4}\right),
$$


with the electric force

$$
f_{e}=-\frac{\partial\left(Q^{2} / 2 C\right)}{\partial(2 \pi R)}=\frac{Q^{2}}{4 \pi^{2} R^{3}}\left(\ln \frac{b}{a}-\frac{3}{4}\right) .
$$

Calculation of the magnetic force in this case shows that, as above, it is equal to the electric one, and the total force is twice the electric force,

$$
f_{\rho}=f_{m}+f_{e}=2 f_{e}=\frac{I^{2}}{R c^{2}}\left(2 \ln \frac{b}{a}-\frac{3}{2}\right) .
$$

The above derivation is very simple, but it does not tell how the centrifugal force varies in the cross section of the beam. To answer this question, we need to find the electric and magnetic fields inside the beam. For the beam in vacuum, we will find these fields using a perturbation theory in small parameter $a / R$.

As a first step in calculations, we need the electrostatic potential $\phi$ and the vector potential $A_{\theta}$ at distances far from the center of the beam in comparison with the beam radius, $r=\sqrt{x^{2}+z^{2}} \gg a$, but close, relative to the orbit size, $R \gg r$. This approximation is equivalent to the limit of infinitely thin beam, $a \rightarrow 0$, and the result can be found in textbooks (see, e.g. [12]),

$$
\begin{gathered}
\phi(\rho, z)=2 \mu \sqrt{\frac{R}{\rho}} \kappa K\left(\kappa^{2}\right), \\
A_{\theta}(\rho, z)=\left(\frac{2}{\kappa^{2}}-1\right) \phi-4 \mu \sqrt{\frac{R}{\rho}} \kappa^{-1} E\left(\kappa^{2}\right),
\end{gathered}
$$

where $\kappa^{2}=4 \rho R /\left[(\rho+R)^{2}+z^{2}\right], \rho$ is the radius counted from the center of the orbit, and $\mu$ is the charge per unit length. Expanding this expression in the vicinity of the beam, $x=\rho-R, r=\sqrt{x^{2}+z^{2}} \ll R$, one finds

$$
\begin{gathered}
\phi=-2 \mu\left(1-\frac{x}{2 R}\right) \ln \frac{\sqrt{x^{2}+z^{2}}}{8 R}+\mu \frac{x}{R}+\ldots, \\
A_{\theta}=\phi-4 \mu\left(1-\frac{x}{2 R}\right)+\ldots
\end{gathered}
$$

To find the potential $\phi$ inside the beam we need to solve the Poisson equation,

$$
\Delta \phi=\frac{1}{\rho} \frac{\partial}{\partial \rho} \rho \frac{\partial \phi}{\partial \rho}+\frac{\partial^{2} \phi}{\partial z^{2}}=-4 \pi e n(\rho, z)
$$

where $n$ is the particle density in the beam. Using the coordinate $x, \rho=R+x, x \ll$ $R$, we can expand the first term in the equation keeping only linear terms in $R^{-1}$, 


$$
\frac{\partial^{2} \phi}{\partial x^{2}}+\frac{\partial^{2} \phi}{\partial z^{2}}=-4 \pi e n-\frac{1}{R} \frac{\partial \phi}{\partial x}+O\left(R^{-2}\right)
$$

Let us assume that $\phi=\phi_{0}+\phi_{1}$, where $\phi_{0}$ is the potential in the limit $R \rightarrow \infty$, and $\phi_{1}$ is linear in $R^{-1}, \phi_{1} \ll \phi_{0}$. In the zeroth approximation, we have

$$
\frac{\partial^{2} \phi_{0}}{\partial x^{2}}+\frac{\partial^{2} \phi_{0}}{\partial z^{2}}=-4 \pi e n
$$

For a constant density beam, $n=$ const for $r<a$, the solution of Eq. (25) is

$$
\begin{aligned}
\phi_{0} & =\mu\left(1-\frac{r^{2}}{a^{2}}\right)+2 \mu \ln \frac{8 R}{a}, \quad r<a, \\
\phi_{0} & =-2 \mu \ln \frac{r}{8 R}, \quad r>a,
\end{aligned}
$$

where $\mu=\pi a^{2}$ en is the beam charge per unit length. In Eq. (26) we took into account that in the region $a \ll r \ll R$ it should match the axisymmetric part of the asymptotic expression $\phi \approx 2 \mu \ln (8 R / r)$ which follows from Eq. (21). In the first order, the potential $\phi_{1}$ satisfies the equation

$$
\frac{\partial^{2} \phi_{1}}{\partial x^{2}}+\frac{\partial^{2} \phi_{1}}{\partial z^{2}}=-\frac{1}{R} \frac{\partial \phi_{0}}{\partial x}
$$

The solution can be found by solving Eq. (27) and using as a boundary condition the asymptotic behaviour for large $r$, given by Eq. (21). The potential $\phi_{1}$ inside the beam is,

$$
\phi_{1}=\frac{\mu}{R} x\left(1+\frac{r^{2}}{4 a^{2}}+\ln \frac{a}{8 R}\right), \quad r<a .
$$

In a similar fashion, we can find the vector potential $A_{\theta}$ that satisfies the equation

$$
\Delta A_{\theta}=-4 \pi e n
$$

but has a different asymptotic condition at large $r$, Eq. (22). The result is

$$
\begin{array}{ll}
A_{\theta 0}=-\mu\left(\frac{r^{2}}{a^{2}}+2 \ln \frac{a}{8 R}-3\right), & r<a, \\
A_{\theta 1}=\frac{\mu}{R} x\left(3+\frac{r^{2}}{4 a^{2}}+\ln \frac{a}{8 R}\right), & r<a .
\end{array}
$$

It is interesting to note, that although $\phi$ and $A_{\theta}$ satisfy the same equation (see Eq. (23), and Eq. (29)), $A_{\theta} \neq \phi$ due to different asymptotic conditions at large $r$.

Using Eqs. (28) and Eq. (30) for the potentials, one can find the fields inside the beam and calculate the distribution of the transverse force over the cross section of the bunch. This force, as a function of radius $r$ is given by the following equation, 


$$
F_{\rho}=\frac{\mu}{R}\left(-1-\frac{r^{2}}{a^{2}}+2 \ln \frac{8 R}{a}\right) .
$$

We see that the force has a parabolic profile with the maximum value on the axis of the beam. Averaging this force over the cross section yields

$$
\bar{F}_{\rho}=\frac{\mu}{R}\left(2 \ln \frac{8 R}{a}-\frac{3}{2}\right) .
$$

To compare this result with Eq. (15), we need to take into account that $\mu=I / c$ and the force per units length of the beam $f_{\rho}$ equals the force per unit charge $F_{\rho}$ multiplied by $I / c$. With those factors, we conclude that both results agree with each other.

Using Eq. (31) and (32) we can also find the relative difference between the force and its average value

$$
\frac{F_{\rho}-\bar{F}_{\rho}}{\bar{F}_{\rho}}=\frac{1-2 r^{2} / a^{2}}{4 \ln (8 R / a)-3} .
$$

For a thin bunch, when $\ln (8 R / a) \gg 1$, the variation of the force in the cross section is relatively small.

At this point, it is instructive to consider the transverse particle motion in a coasting beam under the influence of the centrifugal force. Such motion in the horizontal plane, $z=0$, is governed by the following equation

$$
x^{\prime \prime}+K x=\frac{e F_{\rho}(x)}{E}+\frac{1}{R(s)} \frac{\Delta E}{E},
$$

where $K$ is the external focusing, and $\Delta E$ is the particle energy variation arising due to the potential inside the beam, $\Delta E=-e \phi(x)$,

$$
x^{\prime \prime}+K x=\frac{e}{E}\left(F_{\rho}-\frac{\phi}{R}\right) .
$$

For $z=0$, from Eqs. (26) and (31) we have

$$
\begin{aligned}
\phi & =\mu\left(1-\frac{x^{2}}{a^{2}}\right)+2 \mu \ln \frac{8 R}{a}, \\
F_{\rho} & =\frac{\mu}{R}\left(2 \ln \frac{8 R}{a}-1-\frac{x^{2}}{a^{2}}\right) .
\end{aligned}
$$

We see that $F_{\rho}-\phi / R=-2 \mu / \rho$ does not depend on $x$, which means that the centrifugal force does not contribute to the betatron tune in first order in $R^{-1}$, in agreement with Ref. [9]. The difference $F_{\rho}-\phi / R$ also agrees with the value of the effective centripetal force found in Ref. [10]. 


\section{SHORT BUNCH}

To calculate the transverse force for a short bunch, we will use both LienardWiechert fields and the result found in the previous section for a coasting beam. We will assume that the bunch density is constant in the cross section within the radius $\sigma_{r}=a$, and the longitudinal charge distribution per unit length is given by $\mu(s)$ with the rms bunch length $\sigma_{z}$.

To find the force $F_{\rho}$ acting on unit charge in the bunch at point $s=s_{0}$, we select a small slice of the bunch of length $\Delta s$, such that $\sigma_{z} \gg \Delta s \gg a$, with a local density $\mu\left(s_{0}\right)$, and calculate the contribution to the force separately from the slice and from the rest of the beam. First, let us find the contribution to the force from the bunch excluding the slice. This can be done by integrating the force shown in Fig. 4. Since the force from a particle of charge $q$ located at point $s$ ahead of the point $s_{0}$ is equal to $q / R\left(s-s_{0}\right)$, the contribution from the part of the bunch in front of the slice is

$$
\frac{1}{R} \int_{s_{0}+\Delta s / 2}^{\infty} \frac{\mu\left(s^{\prime}\right) d s^{\prime}}{s-s^{\prime}}=-\frac{\mu\left(s_{0}\right)}{R} \ln \frac{\Delta s}{2 R}-\frac{1}{R} \int_{s_{0}}^{\infty} \ln \left(\frac{s^{\prime}-s}{R}\right) \frac{d \mu\left(s^{\prime}\right)}{d s^{\prime}} d s^{\prime} .
$$

In the second integral, we extended the integration region from $s_{0}$, because $\Delta s$ is small, and the integral converges at the lower limit. Similarly, the force from a particle behind the point $s_{0}$ is equal to $q / 3 R\left(s_{0}-s\right)$, and the contribution from the part of the bunch behind the slice is

$$
\frac{1}{3 R} \int_{-\infty}^{s_{0}-\Delta s / 2} \frac{\mu\left(s^{\prime}\right) d s^{\prime}}{s-s^{\prime}}=-\frac{\mu\left(s_{0}\right)}{3 R} \ln \frac{\Delta s}{2 R}+\frac{1}{3 R} \int_{-\infty}^{s_{0}} \ln \left(\frac{s-s^{\prime}}{R}\right) \frac{d \mu\left(s^{\prime}\right)}{d s^{\prime}} d s^{\prime} .
$$

To find the contribution to the force from the slice itself, we will use the following trick. Consider a circular dc beam of density $\mu=\mu\left(s_{0}\right)$ and select a slice $\Delta s$ with its center located at $\psi=0$. From the previous section, we know that the force in this case does not depend on position and is given by Eq. (32). If we subtract from this force the contribution $\tilde{F}$ of the part of the circle external to the slice, that is the part occupying the region $\Delta \psi / 2<\psi<2 \pi-\Delta \psi / 2$, where $\Delta \psi=\Delta s / R$, we will find the force of the slice itself. The quantity $\tilde{F}$ is equal

$$
\tilde{F}=\int_{\Delta \psi / 2}^{2 \pi-\Delta \psi / 2} F_{\rho}(\psi) d \psi=\int_{\Delta \psi / 2}^{2 \pi-\Delta \psi / 2}\left[E_{\rho}(\psi)+H_{z}(\psi)\right] d \psi
$$

where $E_{\rho}(\psi)$ and $H_{z}(\psi)$ are given by Eqs. (4) and (5) with $q$ substituted by the charge per unit angle $\mu R$. The result of the integration in the limit of small $\Delta s$ is

$$
\tilde{F}=\int_{\Delta \psi / 2}^{2 \pi-\Delta \psi / 2} F_{\rho}(\psi) d \psi=\frac{\mu}{R}\left(A+\frac{4}{3} \ln \frac{R}{\Delta s}\right),
$$

where the constant $A=3.33$ was found from numerical integration. If we now subtract this result from the force of the dc current, Eq. (32), the difference 


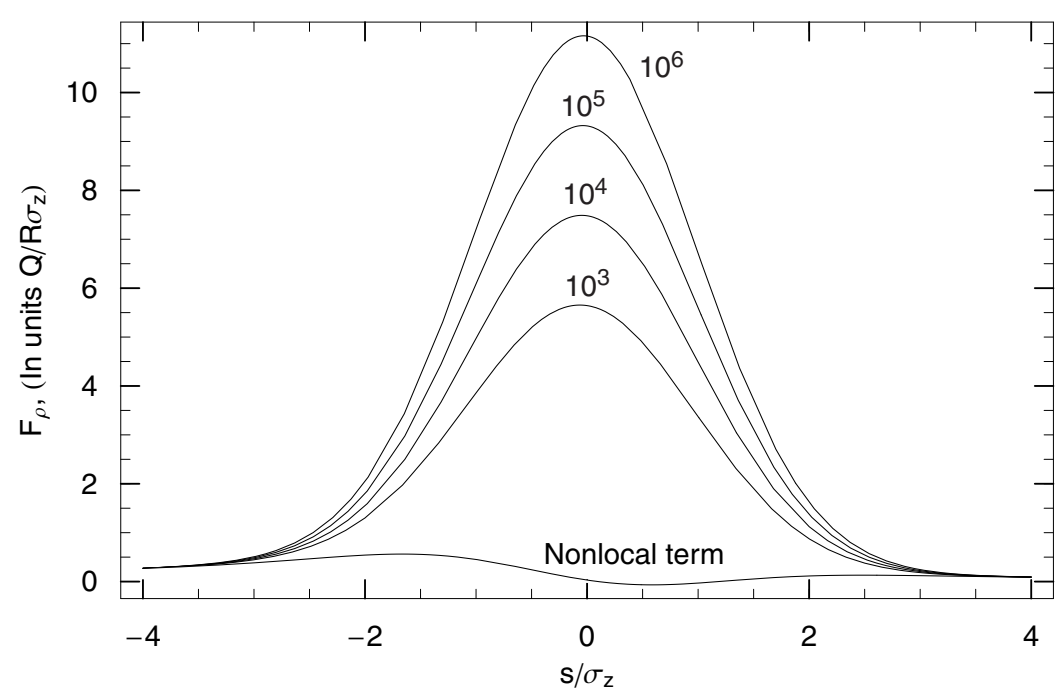

FIGURE 6. Transverse force measured in units $Q / R \sigma_{z}$ for a short Gaussian bunch for several different ratios $R / a$ indicated by a number near the curve. The bottom curve shows the contribution of the second term in Eq. (42) which does not depend on the ratio $R / a$.

$$
\frac{\mu\left(s_{0}\right)}{R}\left(2 \ln \frac{8 R}{a}-\frac{3}{2}\right)-\frac{\mu\left(s_{0}\right)}{R}\left(A+\frac{4}{3} \ln \frac{R}{\Delta s}\right)
$$

is equal to the force induced by the slice at its center.

Summing Eqs. (37), (38) and (41) gives the total transverse force in a short bunch:

$$
\begin{aligned}
\bar{F}_{\rho}(s) & =\frac{\mu(s)}{R}\left(2 \ln \frac{8 R}{a}-3.91\right) \\
& +\frac{1}{R} \int_{0}^{\infty} \ln \xi\left(\frac{1}{3} \mu^{\prime}(s-\xi)-\mu^{\prime}(s+\xi)\right) d \xi
\end{aligned}
$$

As we see from this equation, the first term in this force is local - it depends on the charge density at the observation point. This term also logarithmically depends on the bunch radius $a$. The second term involves the distribution of the charge in the bunch. The plot of the force for a Gaussian bunch for several different ratios $R / a$ is shown in Fig. 6. As we see, for large values of $R / a$, typical in accelerators with short bunches, the dominant contribution comes from the term that is proportional to the local current in the bunch.

\section{NUMERICAL ESTIMATES}

In this section, we estimate the effect of the transverse force on the emittance growth of a bunch passing through a bend and compare it with the emittance growth due to the longitudinal CSR force. We start from the longitudinal CSR 
wake. When the beam passes through the magnet, the energy within the bunch changes, and due to the variation of the energy, the deflection angle $\Delta x^{\prime}$ for different slices of the bunch also varies. This variation is given by the following formula,

$$
\Delta x^{\prime}(s)=\frac{\theta w_{\text {bunch }}(s) N e^{2} L_{b}}{2 E}
$$

where $\theta$ is the deflection angle for the nominal energy, $\theta \approx L_{b} / R, L_{b}$ is the length of the bend, and $E$ is the beam energy. For a short bend, the variance of the deflection angle is proportional to the increase of the projected emittance $\Delta \epsilon_{N}$ (we assume that $\left.\Delta \epsilon_{N} \ll \epsilon_{N}\right)$,

$$
\Delta \epsilon_{N}=\frac{1}{2} \gamma \beta\left\langle\left(\Delta x^{\prime}-\left\langle\Delta x^{\prime}\right\rangle\right)^{2}\right\rangle,
$$

where $\beta$ is the beta function at the location of the bend. For a Gaussian bunch, using Eq. (10), we find

$$
\Delta \epsilon_{N}=7.5 \times 10^{-3} \frac{\beta}{\gamma}\left(\frac{N r_{e} L_{b}^{2}}{R^{5 / 3} \sigma_{z}^{4 / 3}}\right)^{2} .
$$

To estimate the effect numerically, we will use the parameters of one of the magnets of the LCLS bunch compressor [13]: $\theta=3.6^{\circ}, L_{b}=1.5 \mathrm{~m}, E=6 \mathrm{GeV}$, $\sigma_{z}=60 \mu \mathrm{m}, N=6 \times 10^{9}, R=24 \mathrm{~m}, \beta=10 \mathrm{~m}$. Putting these numbers into Eq. (45) gives

$$
\Delta \epsilon_{N}=4.2 \times 10^{-8} \mathrm{~m}
$$

which is about $4 \%$ of the nominal emittance in the LCLS [14].

To estimate the emittance growth due to the transverse wake, we note that the transverse force in the bend deflects the slice by

$$
\Delta x^{\prime}(s)=\frac{1}{E} \bar{F}_{\rho}(s), e L_{b}
$$

where $\bar{F}_{\rho}$ is the centrifugal force averaged over the cross section. Again, using Eq. (44) we find

$$
\Delta \epsilon_{N} \approx 2.5 \times 10^{-2} \frac{\beta}{\gamma}\left(\frac{\Lambda N r_{e} L_{b}}{R \sigma_{z}}\right)^{2}
$$

where $\Lambda$ is a logarithmic factor equal to $\ln (8 R / a)$ if the beam travels in vacuum. To approximately take into account the effect of the walls of the vacuum chamber, we will use for $\Lambda$ the value $\ln (b / a)$ following from Eq. (18). Assuming $b / a=150$, that gives $\Lambda=5$, we find

$$
\Delta \epsilon_{N}=1.6 \times 10^{-7} \mathrm{~m},
$$

that is about four times larger than the longitudinal effect. 


\section{CONCLUSION}

We found that the centrifugal force for a short bunch is approximately given by

$$
\bar{F}_{\rho}(s) \approx \Lambda \frac{\lambda(s)}{R}
$$

where $\Lambda$ is a logarithmic factor, typically of the order of several units. The presence of the conducting walls does not eliminate the transverse force and only modifies the factor $\Lambda$. Although the centrifugal force does not contribute to the tune shift in a circular accelerator, it does effect the transverse motion of the beam. One of the examples, considered in this paper, is the emittance growth of a short bunch in a magnetic compressor. In this case, the ratio of the emittance growth due to the centrifugal force and that due to unshielded CSR wake is of the order

$$
\Lambda^{2}\left(\frac{R}{L_{b}}\right)^{2}\left(\frac{\sigma_{z}}{R}\right)^{2 / 3}
$$

The relative role of the transverse force becomes essential for short bends (although the gross emittance increase goes down with $L_{b}$ ).

\section{ACKNOWLEDGEMENTS}

This work was supported by Department of Energy contract DE-AC03$76 \mathrm{SF} 00515$.

\section{REFERENCES}

1. Iogansen, L. V., and Rabinovich, M. S., Sov. Phys. JETP 37, 83 (1960).

2. Murphy, J. B., Krinsky, S., and Gluckstern, R. L., "Longitudinal Wakefield for Synchrotron Radiation" in Proc. IEEE Particle Accelerator Conference and International Conference on High-Energy Accelerators, Dallas, 1995 (IEEE, Piscataway, NJ, 1996), pp. 2980-2982.

3. Derbenev, Y. S., Rossbach, J., Saldin, E. L., and Shiltsev, V. D., DESY FEL Report TESLA-FEL 95-05, DESY, Hamburg, Germany (unpublished).

4. Murphy, J. B., Krinsky, S., and Gluckstern, R. L., Part. Accel. 57, 9 (1997).

5. Saldin, E. L., Schneidmiller, E. A., and Yurkov, M. V., DESY FEL Report TESLAFEL 96-14, DESY, Hamburg, Germany (unpublished).

6. Saldin, E. L., Schneidmiller, E. A., and Yurkov, M. V., Technical Report No. DESYTESLA-FEL-97-08, DESY, Hamburg, Germany (unpublished).

7. Kheifets, S. A., and Zotter, B., "Shielding effects on coherent synchrotron radiation", in Proc. Micro Bunches Workshop, Upton, 1995, No. 367 in AIP Conference Proceedings, edited by E. B. Blum, M. Dienes, and J. B. Murphy (American Institute of Physics, New York, 1995), pp. 424-434. 
8. Talman, R., Phys. Rev. Lett. 56, 1429 (1986).

9. Lee, E. P., Part. Accel. 25, 241 (1990).

10. Derbenev, Y. S., and Shiltsev, V. D., Technical Report No. SLAC-PUB-7181, SLAC, Stanford, CA, USA (unpublished).

11. Jackson, J. D., Classical Electrodynamics, 2nd ed. (Wiley, New York, 1975).

12. Landau, L. D., and Lifschitz, E. M., Electrodynamics of Continuous Media (Pergamon, NY, 1960).

13. LCLS Design Study Group, Technical Report No. SLAC-R-521, SLAC (unpublished).

14. Emma, P., and Brinkmann, R., Technical Report No. SLAC-PUB-7554, SLAC, Stanford, CA, USA (unpublished). 